Washington University School of Medicine Digital Commons@Becker

Open Access Publications

2020

\title{
Six-year follow-up of a trial of antenatal vitamin D for asthma reduction
}

\author{
Augusto A. Litonjua \\ University of Rochester \\ Vincent J. Carey \\ Harvard University \\ Nancy Laranjo \\ Brigham and Women's Hospital \\ Benjamin J. Stubbs \\ Brigham and Women's Hospital \\ Hooman Mirzakhani \\ Harvard University
}

See next page for additional authors

Follow this and additional works at: https://digitalcommons.wustl.edu/open_access_pubs

Please let us know how this document benefits you.

\author{
Recommended Citation \\ Litonjua, Augusto A.; Carey, Vincent J.; Laranjo, Nancy; Stubbs, Benjamin J.; Mirzakhani, Hooman; \\ O'Connor, George T.; Sandel, Megan; Beigelman, Avraham; Bacharier, Leonard B.; Zeiger, Robert S.; Schatz, \\ Michael; Hollis, Bruce W.; and Weiss, Scott T., "Six-year follow-up of a trial of antenatal vitamin D for \\ asthma reduction." The New England Journal of Medicine. 382, 6. 525 - 533. (2020). \\ https://digitalcommons.wustl.edu/open_access_pubs/8816
}

This Open Access Publication is brought to you for free and open access by Digital Commons@Becker. It has been accepted for inclusion in Open Access Publications by an authorized administrator of Digital Commons@Becker. For more information, please contact vanam@wustl.edu. 


\section{Authors}

Augusto A. Litonjua, Vincent J. Carey, Nancy Laranjo, Benjamin J. Stubbs, Hooman Mirzakhani, George T. O'Connor, Megan Sandel, Avraham Beigelman, Leonard B. Bacharier, Robert S. Zeiger, Michael Schatz, Bruce W. Hollis, and Scott T. Weiss 
ORIGINAL ARTICLE

\section{Six-Year Follow-up of a Trial of Antenatal Vitamin D for Asthma Reduction}

\author{
Augusto A. Litonjua, M.D., M.P.H., Vincent J. Carey, Ph.D., \\ Nancy Laranjo, B.A., Benjamin J. Stubbs, B.A., Hooman Mirzakhani, M.D., Ph.D., \\ George T. O'Connor, M.D., Megan Sandel, M.D., M.P.H., Avraham Beigelman, M.D., \\ Leonard B. Bacharier, M.D., Robert S. Zeiger, M.D., Ph.D., Michael Schatz, M.D., \\ Bruce W. Hollis, Ph.D., and Scott T. Weiss, M.D.
}

\section{A BSTRACT}

\section{BACKGROUND}

We previously reported the results of a trial of prenatal vitamin D supplementation to prevent asthma and recurrent wheeze in young children, which suggested that supplementation provided a protective effect at the age of 3 years. We followed the children through the age of 6 years to determine the course of asthma and recurrent wheeze.

\section{METHODS}

In this follow-up study, investigators and participants remained unaware of the treatment assignments through the children's sixth birthday. We aimed to determine whether, when maternal levels of 25-hydroxyvitamin D were taken into account, children born to mothers who had received $4400 \mathrm{IU}$ of vitamin $\mathrm{D}_{3}$ per day during pregnancy (vitamin D group) would have a lower incidence of asthma and recurrent wheeze at the age of 6 years than would those born to mothers who had received $400 \mathrm{IU}$ of vitamin $\mathrm{D}_{3}$ per day (control group). Time-to-event methods were used to compare the treatment groups with respect to time to the onset of asthma or recurrent wheeze. Multivariate methods were used to compare longitudinal measures of lung function between the treatment groups.

\section{RESULTS}

There was no effect of maternal vitamin D supplementation on asthma and recurrent wheeze in either an intention-to-treat analysis or an analysis with stratification according to the maternal 25-hydroxyvitamin $\mathrm{D}$ level during pregnancy. There was no effect of prenatal vitamin D supplementation on most of the prespecified secondary outcomes. We found no effects of prenatal supplementation on spirometric indexes. Although there was a very small effect on airway resistance as measured by impulse oscillometry, this finding was of uncertain significance.

\section{CONCLUSIONS}

Vitamin D supplementation during the prenatal period alone did not influence the 6-year incidence of asthma and recurrent wheeze among children who were at risk for asthma. (Funded by the National Heart, Lung, and Blood Institute; VDAART ClinicalTrials.gov number, NCT00920621.)
From the Division of Pulmonary Medicine, Department of Pediatrics, Golisano Children's Hospital at Strong, University of Rochester Medical Center, Rochester, NY (A.A.L.); the Channing Division of Network Medicine, Department of Medicine, Brigham and Women's Hospital (V.J.C., N.L., B.J.S., H.M., S.T.W.), Harvard Medical School (V.J.C., H.M., S.T.W.), the Pulmonary Center, Department of Medicine, Boston University School of Medicine (G.T.O.), and the Department of Pediatrics, Boston Medical Center (M. Sandel) - all in Boston; the Division of Allergy, Immunology, and Pulmonary Medicine, Department of Pediatrics, St. Louis Children's Hospital, Washington University School of Medicine, St. Louis (A.B., L.B.B.); Kaiser Permanente Southern California, San Diego (R.S.Z., M. Schatz); and the Department of Pediatrics, Medical University of South Carolina, Charleston (B.W.H.). Address reprint requests to Dr. Litonjua at Golisano Children's Hospital, 601 Elmwood Ave., Rochester, NY 14642, or at augusto_litonjua@ urmc.rochester.edu.

N Engl J Med 2020;382:525-33. DOI: 10.1056/NEJMoa1906137 Copyright @ 2020 Massachusetts Medical Society. 
V

ITAMIN D DEFICIENCY HAS BEEN HYpothesized to have contributed to the increase in asthma and allergies in westernized societies. ${ }^{1}$ Because asthma and wheezing illnesses begin very early in life, studies of prenatal and early-life risk factors are crucial. Observational studies have suggested that a higher maternal vitamin D level is associated with a lower risk of asthma-related outcomes among children, and clinical trials with adequate dosing of vitamin $\mathrm{D}$, therefore, are needed to investigate whether prenatal vitamin D supplementation could prevent the development of asthma.

We conducted the Vitamin D Antenatal Asthma Reduction Trial (VDAART), a trial of prenatal vitamin D supplementation to prevent asthma and wheeze in young children through their third birthday. ${ }^{2}$ We previously reported our results, which suggested a reduction in asthma and recurrent wheeze in 3-year-old children in association with vitamin D supplementation. ${ }^{3}$ Secondary analyses showed that the effect of supplementation was significantly stronger among children born to mothers who had higher vitamin D levels at entry into the trial in early pregnancy, ${ }^{4}$ which suggested that maternal levels are important considerations. A meta-analysis of data from our trial and another trial of prenatal vitamin D supplementation showed a significant protective effect of prenatal supplementation on asthma and recurrent wheeze at 3 years $^{5}$ and confirmed that the effect was strongest in women with an initial 25-hydroxyvitamin D level of at least 30 ng per milliliter (75 nmol per liter).

Since asthma in early life is difficult to ascertain with confidence and early wheezing is probably a conglomerate of different phenotypes, we continued to follow the children in our previously reported clinical trial through their sixth birthday. In this report, we present the results of our follow-up study, in which we examined whether prenatal supplementation with 4400 IU of vitamin $\mathrm{D}_{3}$ or $400 \mathrm{IU}$ of vitamin $\mathrm{D}_{3}$ per day, with maternal levels of 25-hydroxyvitamin $\mathrm{D}$ taken into account, would lead to a decreased incidence of asthma and recurrent wheeze at the age of 6 years.

METHODS

PARTICIPANTS

We recruited pregnant women from three clinical sites across the United States - Boston Medical Center, Boston; Washington University at St. Louis,
St. Louis; and Kaiser Permanente Southern California Region, San Diego. The data coordinating center was based in the Channing Division of Network Medicine, Brigham and Women's Hospital, Boston. Eligible participants were women between the ages of 18 and 39 years who presented when the estimated duration of the ongoing pregnancy was between 10 and 18 weeks; who had a history of asthma, eczema, or allergic rhinitis or whose partner (biologic father of the child) had a history of asthma, eczema, or allergic rhinitis; who was a nonsmoker; and who was English- or Spanish-speaking and intended to participate for 4 years (up to the third birthday of the child). The purpose of this follow-up study was to determine whether the early findings concerning the effect of vitamin D supplementation would extend to the sixth birthday of the child.

The original VDAART trial protocol and the protocol for the follow-up study, available with the full text of this article at NEJM.org, were approved by the institutional review boards at each participating institution. All women provided written informed consent. The authors vouch for the accuracy and completeness of the data and for the fidelity of the study to the protocol.

\section{TRIAL DESIGN}

Details of the trial design and protocol through the age of 3 years have been published previously. ${ }^{2,3}$ VDAART was a randomized, double-blind, placebo-controlled trial of prenatal vitamin D supplementation in which participants received either a daily vitamin D dose of 4000 IU plus a multivitamin with 400 IU of vitamin D (vitamin D group) or daily placebo plus a multivitamin with 400 IU of vitamin D (control group). Participants were screened for eligibility from October 2009 through July 2011. The original research grant that funded the trial was a cooperative agreement with the National Heart, Lung, and Blood Institute (NHLBI) of the National Institutes of Health that lasted for 5 years, and the outcomes were ascertained when the children were 3 years old. ${ }^{3}$ Because of the heterogeneity in the earlylife asthma and wheeze phenotype, the intention was to renew the grant to enable follow-up through the children's sixth birthday and thereby analyze a more definitive phenotype.

The current study followed the children from their third birthday through their sixth birthday. Follow-up of the last child was completed in 
January 2018. Prenatal interval visits, labor and delivery room data collection, and postnatal visits and data collected during these visits have been detailed previously. ${ }^{2,3}$ The participants and investigators remained unaware of the treatmentgroup assignments until after the visit corresponding to each child's sixth birthday.

The primary outcome for this follow-up study was an extension of that in the original analysis - asthma, recurrent wheeze, or both. Asthma was defined as a maternal or caregiver report of physician-diagnosed asthma, and time of onset was defined as the first report of wheezing or the first report of the use of any asthma medication (see the Supplementary Appendix, available at NEJM.org). Recurrent wheeze was defined as maternal or caregiver report of wheeze or the use of any asthma medication in 2 separate years over the first 6 years, and the time of onset was the first report of wheeze or the first report of the use of any asthma medication. Analyses of lung-function measures involved data from children who had completed at least two acceptable spirometry or impulse oscillometry tests and whose mother provided vitamin D results at baseline or during the third trimester.

We prespecified several secondary outcomes, including active asthma, parental report of physician's diagnosis of eczema, parental report of physician's diagnosis of allergic rhinitis, total IgE levels, allergen sensitization (specific IgE to a panel of aeroallergens and food allergens), lower respiratory tract infections, and lungfunction measures. Details of the definitions of these outcomes are provided in the Supplementary Appendix.

Lung function was measured by impulse oscillometry at 4 years of age and yearly after that. Spirometry was performed at the 5- and 6-year visits. Impulse oscillometry and spirometry were performed with the MasterScreen IOS and MasterScreen PFT systems with the Jaeger pneumotach (formerly CareFusion, now Vyaire Medical). Participants were instructed that the children should refrain from using bronchodilator inhalers, syrups, or pills for at least 8 hours before the visit and glucocorticoid inhalers or nebulizers and leukotriene modifiers for 24 hours before the visit. All impulse oscillometry and spirometry results were reviewed by expert reviewers from the Pulmonary Function Laboratory of the University of Wisconsin, Madison, who were unaware of the treatment assignments. Acceptabil- ity and repeatability standards adhered to American Thoracic Society guidelines. For spirometry, acceptability criteria were modified to allow for a minimum expiratory time of 1 second, with a 3-second expiratory time or a plateau in the volume-time curve preferred for acceptability. For analyses, we included tests with at least two acceptable maneuvers.

Blood samples were obtained from mothers at enrollment and at 32 to 38 weeks of gestation. Cord blood was obtained at delivery, and children had blood samples obtained at the ages of 1,3 , and 6 years. Circulating 25-hydroxyvitamin D levels from maternal and child plasma samples were determined with the DiaSorin Liaison chemiluminescence immunoassay, ${ }^{6}$ whereas liquid chromatography-tandem mass spectrometry ${ }^{7}$ was used for cord-blood samples.

\section{STATISTICAL ANALYSIS}

We conducted two prespecified analyses to evaluate the effect of prenatal vitamin D supplementation on asthma risk in children. First, we tested the null hypothesis that there was no treatment effect on the risk of the development of physician-diagnosed asthma or recurrent wheeze by age 6 . This was an intention-to-treat analysis of the primary outcome - that is, first onset of asthma or recurrent wheeze. Second, to enhance the risk model for asthma and recurrent wheeze and the associated hypothesis test, we used stratification according to mean maternal vitamin D concentration in pregnancy, including covariate adjustments. To maximize the use of available information and to accommodate varying times between visits, nonparametric intervalcensored lifetime regression methods were used. ${ }^{8,9}$ For the intention-to-treat analysis, the estimated hazard ratio comparing the low dose (control) with the high dose is accompanied by a P value. For the stratified secondary model, intervalcensored proportional-hazards regression was used to compare event-free time distributions, with adjustment for site, maternal asthma status, and the race and ethnic group of the child; the hazard ratio and $95 \%$ confidence interval are reported for the comparison of the bottom quartile with the top quartile of the mean maternal vitamin D concentration.

For secondary categorical outcomes, differences in relative proportions are reported, and for secondary continuous outcomes, mean differences are reported. No adjustments were made 
for multiple comparisons. For longitudinal measures of lung function, marginal means and confidence intervals are reported for each treatment group, and linear mixed-effects modeling was used to produce summarized group-specific, covariate-adjusted estimates and confidence intervals. ${ }^{10}$

\section{RESULTS}

\section{PARTICIPANTS}

The flow of participants is shown in Figure 1, and selected baseline characteristics of the participants are shown in Table 1 and in Table S1 in the Supplementary Appendix. A total of 806 children were included in the analysis. There were no large differences in baseline characteristics. Acceptable lung-function measurements were completed for a subpopulation of participants, and their characteristics are shown in Table S2. The characteristics of the participants in this subpopulation reflect the characteristics of those in the overall trial population. The cordblood levels of 25-hydroxyvitamin D in children born to mothers in the vitamin $\mathrm{D}$ group were significantly higher than those in children born to mothers in the control group, as was previously reported. Subsequent levels at the ages of 1,3 , and 6 years remained low and did not differ significantly between the groups (Fig. S1).

\section{SUPPLEMENTATION, MATERNAL} 25-HYDROXYVITAMIN D LEVELS, AND CHILDHOOD OUTCOMES

By the age of 6 years, a total of 360 children $(44.7 \%)-176(43.5 \%)$ in the vitamin D group and $184(45.9 \%)$ in the control group - met the criteria for the outcome of asthma or recurrent wheeze. Of these 360 children, 198 (55.0\%) had a doctor's diagnosis of asthma, and 344 (95.6\%) had recurrent wheezing.

There was no significant effect of maternal supplementation with 4400 IU of vitamin D per day on the incidence of asthma or recurrent wheeze at the age of 6 years (184 events among 401 participants in the control group and 176 events among 405 participants in the vitamin D group; interval-censored hazard ratio [control vs. vitamin $\mathrm{D}$ ], 1.12; $\mathrm{P}=0.25$ ) (Fig. 2). When event times were stratified according to quartile of mean prenatal level of vitamin D recorded in the mother's serum, an effect was found, with a higher risk among children born to mothers with 25-hydroxyvitamin D levels of less than 20 ng per milliliter (50 nmol per liter) (Fig. S2). An adjusted estimate of the hazard ratio among children born to mothers in the top quartile of mean prenatal 25-hydroxyvitamin D level ( $>35 \mathrm{ng}$ per milliliter; 76 events among 105 participants) as compared with the bottom quartile ( $\leq 20 \mathrm{ng}$ per milliliter; 110 events among 200 participants) was $0.74(95 \%$ confidence interval [CI], 0.54 to 1.02 ).

There were no significant effects of maternal vitamin D supplementation on the secondary outcomes of eczema, allergic rhinitis, or lower respiratory tract infection by 6 years of age (Table 2). Among children born to mothers in the vitamin $\mathrm{D}$ group, the mean $( \pm \mathrm{SD})$ log-transformed total IgE level was 3.91 $\pm 1.64 \log \mathrm{kU}$ per liter, and that among children born to mothers in the control group was $4.22 \pm 1.70 \log \mathrm{kU}$ per liter (mean difference, $-0.31 \log \mathrm{kU}$ per liter; $95 \%$ CI, -0.64 to 0.01 ) (Table 2). Although the number of children who were sensitized to any allergen from the panel of 13 allergens did not differ significantly between the groups, children who were born to mothers in the vitamin $D$ group had fewer positive results of specific IgE tests at 6 years of age than did children born to mothers in the control group (Table 2).

The effects of prenatal supplementation on childhood lung function were measured by impulse oscillometry and spirometry. In models adjusted for race and ethnic group, clinical center, and sex of the child, total airway resistance (resistance at $5 \mathrm{~Hz}$ [R5]) and lower peripheral airway resistance (resistance at $5 \mathrm{~Hz}$ minus resistance at $20 \mathrm{~Hz}$ [R5-R20]) were lower in the vitamin $\mathrm{D}$ group than in the control group among children 5 to 6 years of age (Table S3). There was no association of prenatal vitamin D supplementation with spirometric indexes.

\section{PRENATAL 25-HYDROXYVITAMIN D LEVELS AND CHILDHOOD LUNG FUNCTION}

Because baseline levels may affect the response to supplementation, we investigated the effect of maternal prenatal 25-hydroxyvitamin D levels on the same lung-function measures. Among six such secondary comparisons, we found that children born to mothers with mean 25-hydroxy- 


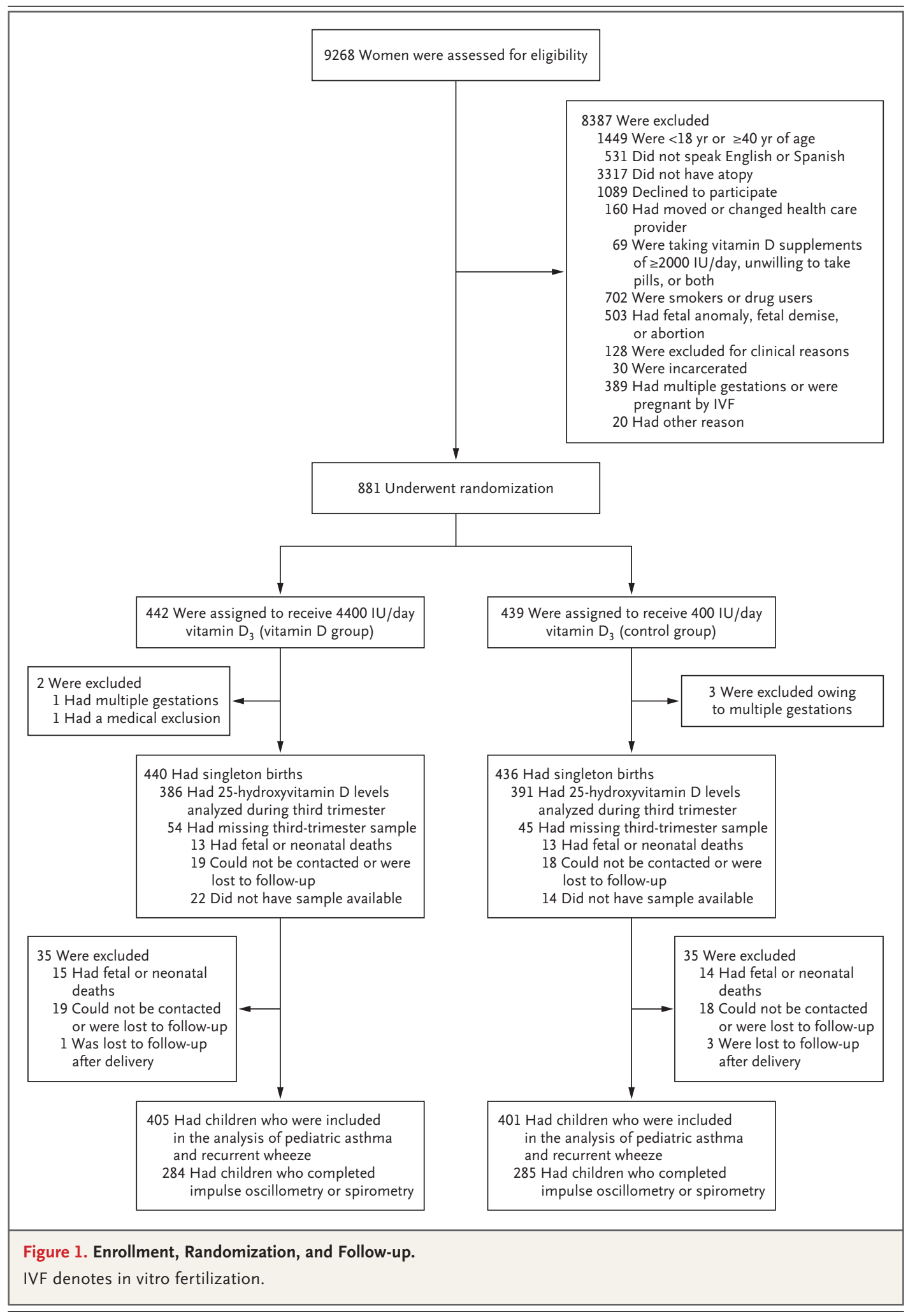

N ENGLJ MED 382;6 NEJM.ORG FEBRUARY 6, 2020

The New England Journal of Medicine

Downloaded from nejm.org at Washington University in St. Louis Becker Library on February 23, 2020. For personal use only. No other uses without permission. Copyright @ 2020 Massachusetts Medical Society. All rights reserved. 


\begin{tabular}{|c|c|c|c|}
\hline Characteristic & $\begin{array}{c}\text { Vitamin D } \\
(\mathrm{N}=405)\end{array}$ & $\begin{array}{l}\text { Control } \\
(\mathrm{N}=401)\end{array}$ & $\begin{array}{c}\text { Overall } \\
(N=806)\end{array}$ \\
\hline Maternal age $-y r$ & $27.5 \pm 5.5$ & $27.2 \pm 5.5$ & $27.3 \pm 5.5$ \\
\hline Gestational age at enrollment $-w k$ & $14.1 \pm 2.8$ & $14.2 \pm 2.7$ & $14.2 \pm 2.8$ \\
\hline Gestational age at delivery — wk & $39.0 \pm 1.9$ & $39.0 \pm 2.0$ & $39.0 \pm 1.9$ \\
\hline Baseline maternal 25-hydroxyvitamin D level - $\mathrm{ng} / \mathrm{ml}$ & $23.3 \pm 10.3$ & $22.6 \pm 10.2$ & $23.0 \pm 10.2$ \\
\hline \multicolumn{4}{|l|}{ Medical history of mother — no. (\%) } \\
\hline History of asthma & $171(42.2)$ & $151(37.7)$ & $322(40.0)$ \\
\hline History of hay fever & $256(63.2)$ & $260(64.8)$ & $516(64.0)$ \\
\hline History of eczema & $126(31.1)$ & $131(32.7)$ & 257 (31.9) \\
\hline \multicolumn{4}{|l|}{ Medical history of father — no. (\%) } \\
\hline History of asthma & $100(24.7)$ & $87(21.7)$ & $187(23.2)$ \\
\hline History of hay fever & $164(40.5)$ & $180(44.9)$ & $344(42.7)$ \\
\hline History of eczema & $77(19.0)$ & $56(14.0)$ & $133(16.5)$ \\
\hline Male sex of child - no. (\%) & $200(49.4)$ & $220(54.9)$ & $420(52.1)$ \\
\hline \multicolumn{4}{|l|}{ Race and ethnic group of the child — no. (\%) $\dagger$} \\
\hline Black, Hispanic or non-Hispanic & $175(43.2)$ & $172(42.9)$ & $347(43.1)$ \\
\hline White, Hispanic & $55(13.6)$ & $57(14.2)$ & $112(13.9)$ \\
\hline White, non-Hispanic & $106(26.2)$ & $108(26.9)$ & $214(26.6)$ \\
\hline Other & $69(17.0)$ & $64(16.0)$ & $133(16.5)$ \\
\hline
\end{tabular}

* Plus-minus values are means \pm SD. Percentages may not total 100 because of rounding. To convert the values for 25-hydroxyvitamin D to nanomoles per liter, multiply by 2.496 .

$\uparrow$ Race and ethnic group were based on information reported by the mother about her own race and ethnic group and about those of the biologic father.

vitamin D levels of less than 30 ng per milliliter differed from those born to mothers with higher 25-hydroxyvitamin D levels in total airway resistance (adjusted mean R5, $1.01 \mathrm{kPa}$ per liter per second [95\% CI, 0.99 to 1.03 ] vs. $0.96 \mathrm{kPa}$ per liter per second [95\% CI, 0.93 to 0.99$]$ ) and peripheral airway resistance (adjusted mean R5R20, $0.35 \mathrm{kPa}$ per liter per second [95\% CI, 0.33 to 0.36$]$ vs. $0.32 \mathrm{kPa}$ per liter per second [95\% CI, 0.30 to 0.34$]$ ), forced expiratory volume in 1 second $\left(\mathrm{FEV}_{1}\right)$ (adjusted mean, 0.97 liters $[95 \%$ CI, 0.94 to 0.99 ] vs. 1.00 liter [ $95 \% \mathrm{CI}, 0.97$ to 1.04]), and forced vital capacity (FVC) (adjusted mean, 1.06 liters [95\% CI, 1.04 to 1.09] vs. 1.12 liters [95\% CI, 1.09 to 1.15]) (Fig. 3).

\section{DISCUSSION}

Supplementation with vitamin D during the prenatal period alone did not prevent the development of asthma or recurrent wheeze in offspring through the age of 6 years. Although a between- group difference was suggested at early time points through the age of 3 years, this effect was not sustained through the age of 6 years. Most of the secondary analyses were also null, although there was a small but uncertain effect of prenatal supplementation on total and peripheral airway resistance as measured by impulse oscillometry. Analyses according to mean prenatal vitamin D level showed that higher levels were associated with lower airway resistance and better spirometric lung-function indexes.

Vitamin D has pleiotropic effects in utero and postnatally, particularly with respect to lung development ${ }^{11}$ and immune function related to asthma. ${ }^{12}$ Studies in murine models have established that vitamin $\mathrm{D}$ deficiency during pregnancy leads to alveolar simplification and impaired airway development in offspring. ${ }^{13}$ Prenatal vitamin D supplementation abolishes most of the detrimental lung effects in the offspring of vitamin D-deficient dams. ${ }^{14}$ A previously published meta-analysis of our results and the results of a 
Danish trial showed that prenatal supplementation had a significant effect in preventing asthma or recurrent wheeze through the age of 3 years. ${ }^{5}$ However, our results and a recently published follow-up study of the Danish trial ${ }^{15}$ show that prenatal supplementation alone is not sufficient to prevent childhood asthma through midchildhood.

Diagnosing asthma in early life is, at best, difficult. Asthma in early childhood differs from asthma in later childhood or adulthood. ${ }^{16}$ Assessments of wheezing phenotypes in several cohorts have shown that some children may wheeze early in life and not later, whereas others may have either persistent wheeze or late-onset wheeze. ${ }^{17}$ Thus, children who wheeze in early childhood may have remission of their symptoms by the time they reach school age. Although there have been attempts to predict which children with early wheezing will go on to have asthma in later life, ${ }^{17,18}$ none of these have proved to be very accurate. This can be seen in our study, in which only fair-to-moderate correspondence was found between the children who had the outcome at 3 years and those who had the outcome at 6 years (Cohen's kappa, 0.51). It is possible that prenatal vitamin D supplementation had an early effect on only a minority of the wheezing phenotypes in early childhood. Thus, these different phenotypes of early wheezing probably have different causes, and combinations of different types of interventions may be needed to more fully prevent the development of asthma by school age and adolescence.

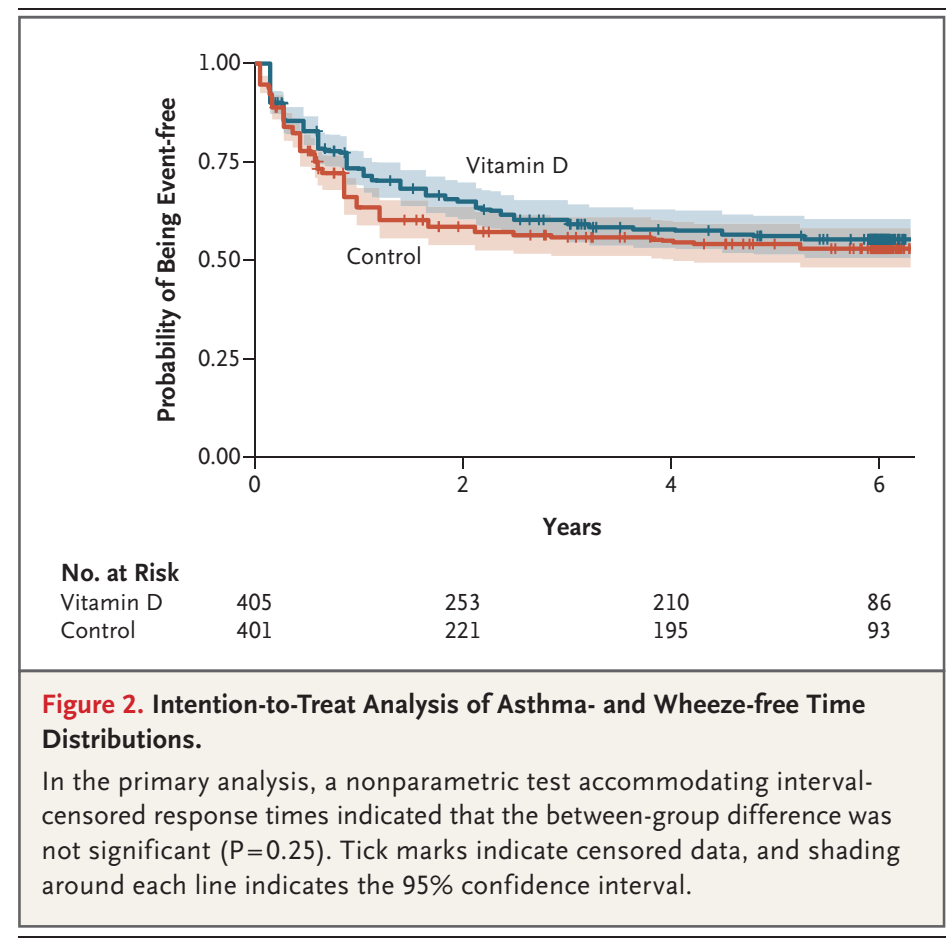

Viral infections are common in early childhood and cause many of the wheeze episodes in this period of life. Given the actions of vitamin D on antimicrobial peptides ${ }^{19,20}$ and virus-induced inflammation, ${ }^{21}$ prenatal vitamin D supplementation may have prevented some of the specific phenotypes of virus-induced wheezing in early life. ${ }^{22}$ Since we did not collect nasal samples during wheezing episodes, we are unable to make further statements regarding virus-induced wheeze

\begin{tabular}{|c|c|c|c|}
\hline Outcome & Vitamin D & Control & Effect $(95 \% \mathrm{Cl}) *$ \\
\hline Active asthma - no./total no. (\%) & $74 / 361(20.5)$ & $59 / 346(17.1)$ & $3.5(-2.5$ to 9.5$)$ \\
\hline $\begin{array}{l}\text { Recurrent wheeze without asthma diagnosis } \\
\quad \text { - no./total no. (\%) }\end{array}$ & $40 / 361(11.1)$ & $55 / 346(15.9)$ & $-4.8(-10.0$ to 0.5$)$ \\
\hline Late-onset wheeze — no./total no. (\%) & $13 / 361(3.6)$ & $14 / 346(4.0)$ & $-0.4(-3.6$ to 4.0$)$ \\
\hline Eczema with typical rash — no./total no. (\%) & $96 / 361(26.6)$ & $91 / 346(26.3)$ & $0.2(-6.4$ to 7.1$)$ \\
\hline Allergic rhinitis - no./total no. (\%) & $131 / 361(36.3)$ & $143 / 346(41.3)$ & $-5.1(-12.5$ to 2.4$)$ \\
\hline Lower respiratory tract infection — no./total no. (\%) & $155 / 361(42.9)$ & $157 / 346(45.4)$ & $-2.4(-10.0$ to 5.1$)$ \\
\hline Total log IgE level — log kU/liter & $3.91 \pm 1.64$ & $4.22 \pm 1.70$ & $-0.31(-0.64$ to 0.01$)$ \\
\hline Any allergic sensitization — no./total no. (\%) & $127 / 213(59.6)$ & $123 / 201(61.2)$ & $-1.6(-11.0$ to 8.0$)$ \\
\hline Percentage of 13 specific IgE tests with positive results & 15 & 18 & $0.82(0.71$ to 0.95$)$ \\
\hline
\end{tabular}

* For outcomes reported as numbers and percentages of participants, the effect is given as the percentage-point difference between the groups. For the percentage of positive results on specific IgE testing, the effect is given as the odds ratio. 


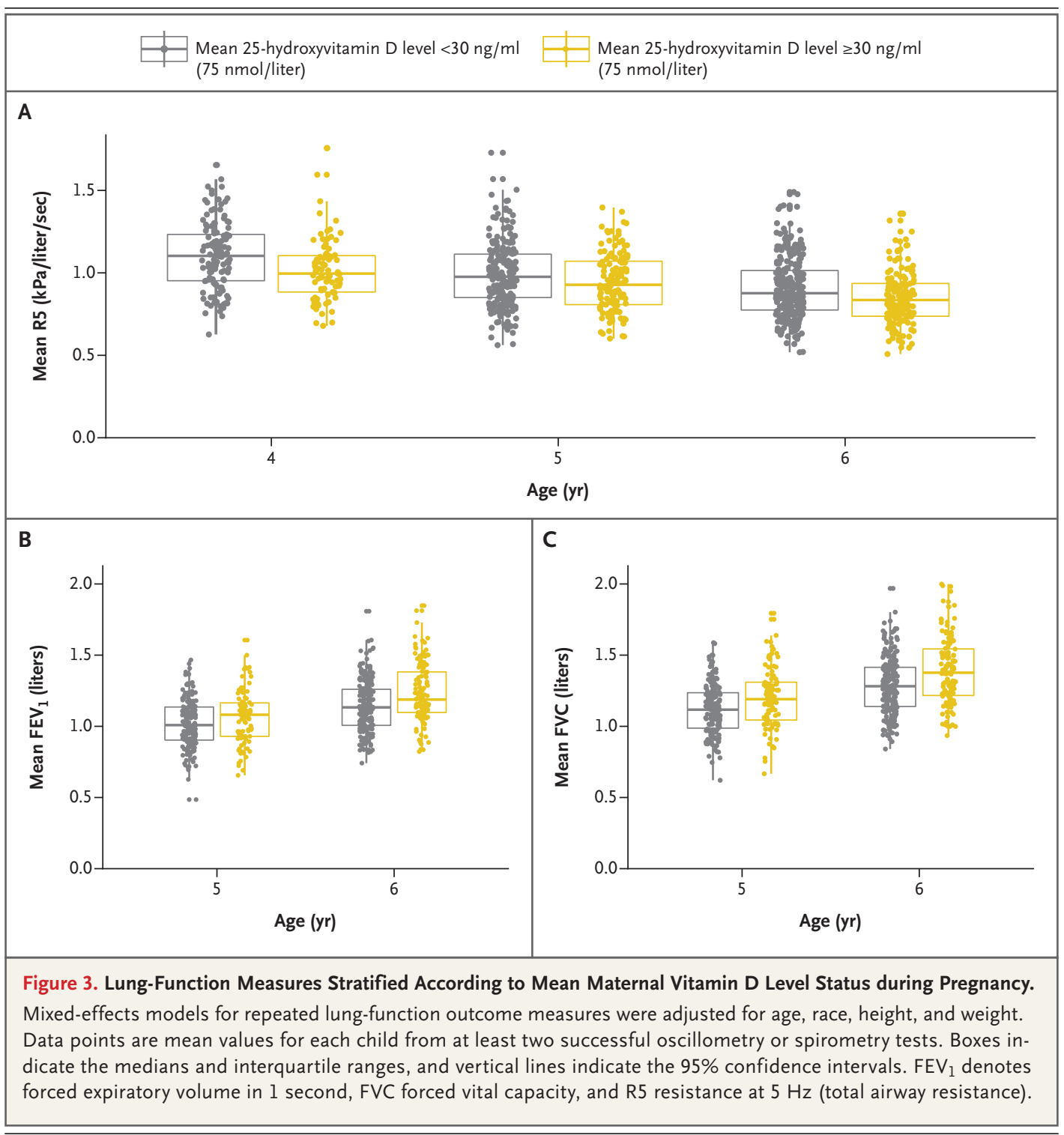

in our study. Another possible explanation for the early wheeze prevention and the absence of later wheeze and asthma prevention is that postnatal supplementation to maintain sufficient vitamin D levels in children may be needed in addition to the prenatal supplementation for sustained effect. ${ }^{23,24}$ These issues will need to be addressed in future studies.

In contrast to the findings of asthma and recurrent wheeze, airway-resistance measures obtained from impulse oscillometry were lower among children born to mothers in the vitamin D group than among children born to mothers in the control group (Table S2). However, these effects were small and will need to be validated in future studies. No treatment effect was seen in the traditional spirometric indexes of $\mathrm{FEV}_{1}, \mathrm{FVC}$, and the $\mathrm{FEV}_{1}$ :FVC ratio.

Our trial has several limitations. First, because postnatal supplementation was not undertaken, we are unable to make any conclusions about the combined effects of prenatal and postnatal supplementation on asthma and recurrent wheeze. As suggested by the recently published D-Wheeze study, ${ }^{23}$ postnatal supplementation has effects in preventing recurrent wheeze. Furthermore, the differences in vitamin D levels noted between the treatment groups in maternal and cord blood were not sustained after birth, possibly contributing to the lack of effect by the age 
of 6 years. Next, women were enrolled in the trial without regard to their initial vitamin $\mathrm{D}$ level, and this is likely to have played a role in biasing our results toward the null. Although our previous secondary analyses suggested that higher maternal levels early in pregnancy were beneficial, ${ }^{4}$ a better-designed trial is needed. The previously published results of our 3-year outcomes, in which the largest effect of supplementation was seen in the children born to the mothers with higher vitamin $\mathrm{D}$ levels early in pregnancy, may seem counterintuitive, since supplementation is thought to benefit those who have a deficiency of the nutrient. ${ }^{25}$ However, since human lung development starts at approximately 3 to 4 weeks of gestation, ${ }^{26}$ early vitamin D levels may be important in this process.
Prenatal vitamin D supplementation alone did not affect the development of asthma and recurrent wheeze in young children through the age of 6 years. The effect of prenatal supplementation on airway resistance through the age of 6 years suggests that there may be prenatal programming of lung airways, but these small effects will need to be validated in future studies.

Supported by a grant (RO1 HL091528) from the National Heart, Lung, and Blood Institute, National Institutes of Health.

Disclosure forms provided by the authors are available with the full text of this article at NEJM.org.

A data sharing statement provided by the authors is available with the full text of this article at NEJM.org.

We thank all the participants in the trial, Mary-Jo BiebleYahnke and Peggy Zingler for reviewing and ensuring the quality of the impulse oscillometric and spirometric results, and the trial coordinators and research assistants for their dedication in their work with the trial participants.

\section{REFERENCES}

1. Litonjua AA, Weiss ST. Is vitamin D deficiency to blame for the asthma epidemic? J Allergy Clin Immunol 2007;120 1031-5.

2. Litonjua AA, Lange NE, Carey VJ, et al. The Vitamin D Antenatal Asthma Reduction Trial (VDAART): rationale, design, and methods of a randomized, controlled trial of vitamin D supplementation in pregnancy for the primary prevention of asthma and allergies in children. Contemp Clin Trials 2014;38:37-50.

3. Litonjua AA, Carey VJ, Laranjo N, et al. Effect of prenatal supplementation with vitamin D on asthma or recurrent wheezing in offspring by age 3 years: the VDAART randomized clinical trial. JAMA 2016;315:362-70.

4. Wolsk HM, Harshfield BJ, Laranjo N, et al. Vitamin D supplementation in pregnancy, prenatal 25(OH)D levels, race, and subsequent asthma or recurrent wheeze in offspring: secondary analyses from the Vitamin D Antenatal Asthma Reduction Trial. J Allergy Clin Immunol 2017;140(5): 1423-1429.e5.

5. Wolsk HM, Chawes BL, Litonjua AA, et al. Prenatal vitamin D supplementation reduces risk of asthma/recurrent wheeze in early childhood: a combined analysis of two randomized controlled trials. PLoS One 2017;12(10):e0186657.

6. Ersfeld DL, Rao DS, Body JJ, et al. Analytical and clinical validation of the $25 \mathrm{OH}$ vitamin D assay for the LIAISON automated analyzer. Clin Biochem 2004; 37:867-74.

7. Grebe SK, Singh RJ. LC-MS/MS in the clinical laboratory - where to from here? Clin Biochem Rev 2011;32:5-31.

8. Delord M, Genin E. Multiple imputation for competing risks regression with interval-censored data. J Stat Comput Simul 2016;86:2217-28.

9. Pan W. A two-sample test with interval censored data via multiple imputation. Stat Med 2000;19:1-11.

10. Pinheiro JC, Bates DM. Mixed-effects models in S and S-PLUS. New York: Springer-Verlag, 2000.

11. Lykkedegn S, Sorensen GL, BeckNielsen SS, Christesen HT. The impact of vitamin $\mathrm{D}$ on fetal and neonatal lung maturation: a systematic review. Am J Physiol Lung Cell Mol Physiol 2015;308: L587-L602.

12. Pfeffer PE, Hawrylowicz CM. Vitamin $\mathrm{D}$ in asthma: mechanisms of action and considerations for clinical trials. Chest 2018;153:1229-39.

13. Saadoon A, Ambalavanan N, Zinn K, et al. Effect of prenatal versus postnatal vitamin D deficiency on pulmonary structure and function in mice. Am J Respir Cell Mol Biol 2017;56:383-92.

14. Yurt M, Liu J, Sakurai R, et al. Vitamin D supplementation blocks pulmonary structural and functional changes in a rat model of perinatal vitamin D deficiency. Am J Physiol Lung Cell Mol Physiol 2014; 307:L859-L867.

15. Brustad N, Eliasen AU, Stokholm J, $\mathrm{B} \phi$ nnelykke $\mathrm{K}$, Bisgaard $\mathrm{H}$, Chawes BL. High-dose vitamin D supplementation during pregnancy and asthma in offspring at the age of 6 years. JAMA 2019;321:1003-5. 16. Trivedi M, Denton E. Asthma in children and adults - what are the differences and what can they tell us about asthma? Front Pediatr 2019;7:256.

17. Kwong CG, Bacharier LB. Phenotypes of wheezing and asthma in preschool children. Curr Opin Allergy Clin Immunol 2019;19:148-53.
18. Oksel C, Granell R, Haider S, et al. Distinguishing wheezing phenotypes from infancy to adolescence: a pooled analysis of five birth cohorts. Ann Am Thorac Soc 2019;16:868-76.

19. Liu PT, Stenger S, Li H, et al. Toll-like receptor triggering of a vitamin D-mediated human antimicrobial response. Science 2006;311:1770-3.

20. Sousa FH, Casanova V, Findlay F, et al. Cathelicidins display conserved direct antiviral activity towards rhinovirus. Peptides 2017;95:76-83.

21. Hansdottir S, Monick MM, Lovan N, Powers L, Gerke A, Hunninghake GW. Vitamin D decreases respiratory syncytial virus induction of NF-kappaB-linked chemokines and cytokines in airway epithelium while maintaining the antiviral state. J Immunol 2010;184:965-74.

22. Stelmach I, Majak P, Jerzynska J, et al. Cord serum 25-hydroxyvitamin D correlates with early childhood viral-induced wheezing. Respir Med 2015;109:38-43.

23. Hibbs AM, Ross K, Kerns LA, et al. Effect of vitamin D supplementation on recurrent wheezing in black infants who were born preterm: the D-Wheeze randomized clinical trial. JAMA 2018;319: 2086-94.

24. Hollams EM, Teo SM, Kusel M, et al. Vitamin D over the first decade and susceptibility to childhood allergy and asthma. J Allergy Clin Immunol 2017;139(2): 472-481.e9.

25. Heaney RP. Guidelines for optimizing design and analysis of clinical studies of nutrient effects. Nutr Rev 2014;72:48-54.

26. Chen L, Zosky GR. Lung development. Photochem Photobiol Sci 2017;16: 339-46.

Copyright (c) 2020 Massachusetts Medical Society. 